\title{
A Gender Comparison of Postobstructive Injury in the Rat Kidney
}

\author{
Mark R. Quinlan ${ }^{a, b}$ Peter Cronin ${ }^{b}$ Padraig J. Daly ${ }^{a, b}$ Ronald W.G. Watson ${ }^{a, b}$ \\ Walter Manucha ${ }^{\mathrm{e}}$ Neil G. Docherty ${ }^{\mathrm{c}}$ John M. Fitzpatrick ${ }^{\mathrm{a}, \mathrm{b}, \mathrm{d}}$ \\ ${ }^{a}$ The Conway Institute of Biomolecular and Biomedical Sciences and ${ }^{b}$ UCD School of Medicine and Medical \\ Sciences, University College Dublin, Belfield, ' Department of Physiology, Trinity College Dublin, and d Division of \\ Surgery, Mater Misericordiae University Hospital, Dublin, Republic of Ireland; érea de Fisiología Patológica, \\ Departamento de Patología, Facultad de Ciencias Médicas, Universidad Nacional de Cuyo, Centro Universitario, \\ Mendoza, Argentina
}

\section{Key Words}

Ureteral obstruction • Apoptosis · Diuresis · Aquaporin

\begin{abstract}
Background/Aims: Unilateral ureteral obstruction (UUO) results in renal injury. Studies report increased injury indices in male rats following UUO. Our study examined whether this gender-based renal response to UUO was reflected in sustained differences following relief of obstruction. Methods: Adult male/female rats (200-400 g) were subjected to either sham surgery (S/RN) or UUO (UUO/RN). At 24 h, obstruction was relieved and all animals underwent contralateral nephrectomy. Five days after initial surgery, animals were placed in metabolic cages and given water ad libitum for $24 \mathrm{~h}$ followed by a 24-hour period of complete water restriction. On day 7, animals were euthanized and samples harvested. Tubular injury, urinary volume/osmolality, creatinine clearance, plasma arginine vasopressin, renal medullary V2 receptor and aquaporin 2 (AQP2) expression were measured. Results: Male UUO/RN rats showed increased renal apoptotic injury and reduced creatinine clearance rates (glomerular filtration rate) vs. females. No gender-dependent differences were observed in urinary osmolality or concentrating ability. AQP2 expression increased post-obstruction. Conclusion: Increased injury in males following UUO remains
\end{abstract}

manifest during early recovery after release of obstruction. Despite this, the grade of postobstructive diuresis is not significantly altered between sexes. This may reflect reduced glomerular filtration rate and elevated AQP2 in male rats.

Copyright $\odot 2010$ S. Karger AG, Basel

\section{Introduction}

Damage to the kidney proximal to the site of obstruction in unilateral ureteral obstruction (UUO) varies in severity according to the duration, location and grade of obstruction.

Ureteric hypercontractility occurs early after obstruction $[1,2]$ and is followed by the progressive pooling of hypotonic urine [3]. Both of these mechanical stress stimuli are linked to the induction of apoptosis and inflammation and may give rise to reductions in renal perfusion and the establishment of tubulointerstitial fibrosis $[4,5]$.

Normal tubular function has been shown to take up to 1 month to recover following a transient UUO of 24-hour duration in rats [6]. Studies in rodents reveal that during obstruction there is a downregulation of expression of

N.G.D. and J.M.F. contributed equally as senior authors.

\section{KARGER \\ Fax +4161306 1234 E-Mail karger@karger.ch} www.karger.com

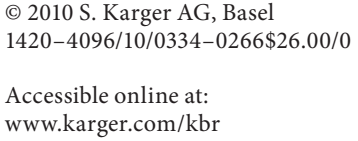

Dr. Neil G. Docherty

Department of Physiology, School of Medicine

University of Dublin, Trinity College

Dublin 2 (Republic of Ireland)

Tel. +353 1896 2076, Fax +3531679 3545, E-Mail dochertn@tcd.ie 
apical sodium channels [7], arginine-vasopressin (AVP)regulated and unregulated aquaporin (AQP) channels [8] and urea transporters [9], events which synergize to cause a functional reduction in collecting duct-free water $\left(\mathrm{H}_{2} \mathrm{O}\right)$ reabsorption and an associated dissipation of the corticomedullary solute gradient. These events underpin the clinical phenomenon of postobstructive diuresis.

A gender bias towards increased acute and chronic renal injury in males has been demonstrated [10]. It is likely that a pro-injury effect of androgens in males (via the activation of local renin-angiotensin, RA, pathways) or a female protective effect secondary to estrogen-induced nitric oxide-dependent cytoprotection at least partly explains this phenomenon.

In models of acute renal injury, male gender is associated with increased severity of numerous indices of injury (apoptosis, inflammation and fibrosis) [11]. The incidence and progression of chronic allograft nephropathy, in which ischemic time is a key risk factor, is also increased in male rats and depends on intrinsic renal production of dihydrotestosterone [12].

Metcalfe et al. [13] recently reported an increase in both proapoptotic and profibrotic indices in male rats associated with a relative worsening in glomerular filtration rate (GFR) following 3 days of UUO. This correlated with testosterone-dependent increases in renal tumor necrosis factor- $\alpha$.

This present study examined whether the dimorphic gender-based response to UUO reported by Metcalfe et al. [13] would result in sustained differences in renal injury and function within 1 week of the relief of UUO of 24 -hour duration in the rat. The chronology of the model was chosen to reflect a time point representative of a clinically relevant time to relief in cases of acute onset obstruction in humans. Creatinine clearance and tubular apoptosis in the postobstructive kidney were examined. Postobstructive diuresis was also examined by measuring urinary concentrating capacity (volume and osmolality before and after a 24-hour period of $\mathrm{H}_{2} \mathrm{O}$ restriction). Finally, plasma AVP levels and the medullary expression of AQP2 and the AVP type 2 receptor (V2R) were compared between male and female rats.

\section{Materials and Methods}

\section{Reagents}

All reagents used in this study were obtained from SigmaAldrich (USA) except those listed below: anti-rabbit IgG HRPlinked antibody and cleaved caspase- 3 antibody (Cell Signaling Technology, USA), enhanced chemiluminescent detection re- agent (Roche Ireland Pharmaceuticals), SuperX X-ray film (Kodak, Ireland), protein assay reagents, kaleidoscope prestained protein standards and PCR reagents (all Bio-Rad Laboratories, UK), creatinine assay kit and arginine vasopressin-ELISA (Cayman Chemical Company, USA), ApopTag kit and Immobilon-P membrane (Millipore, UK), cDNA reagents (Invitrogen, Ireland) and PCR primers (Eurofins, UK).

\section{Animals and Surgery}

All experiments were carried out under a Department of Health and Children license (B100/3611). Adult male and female Wistar rats weighing approximately 200-400 g (2-3 months of age) were anesthetized using isoflurane and subjected to either sham laparotomy or complete obstruction of the left ureter on day 0 . Sham surgery $(\mathrm{S} / \mathrm{RN})$ consisted of a laparotomy during which the left ureter was just identified and manipulated. For obstructed animals (UUO/RN), the mid-ureter was occluded using a plastic, microvascular 'bulldog' clamp. All animals in both groups underwent a right-sided nephrectomy $24 \mathrm{~h}$ later - for the group previously obstructed, the clamps were removed at the time of the contralateral nephrectomy.

\section{Metabolic Cage Studies}

Rats were acclimatized in standard metabolic cages for $48 \mathrm{~h}$ 1 week prior to the initiation of the experimental protocols. On day 4 after nephrectomy (day 5 of experimental protocol) rats were placed in metabolic cages for $24 \mathrm{~h}$ with free access to $\mathrm{H}_{2} \mathrm{O}$ and the urine excreted over that time period was collected. Rats were then completely $\mathrm{H}_{2} \mathrm{O}$ deprived for a further $24 \mathrm{~h}$ and again 24-hour urine was collected.

\section{Sample Collection}

Urine was collected, centrifuged at 10,000 rpm and stored at $-80^{\circ} \mathrm{C}$. Prior to euthanasia (day 7), rats were anesthetized using isoflurane, and following laparotomy a heparinized arterial blood sample was taken from the abdominal aorta at the level of the iliac bifurcation. Blood samples were centrifuged at 3,000 $\mathrm{g}$ for $5 \mathrm{~min}$ and plasma retrieved for storage at $-80^{\circ} \mathrm{C}$. Immediately subsequent to blood sampling, animals were perfused in a retrograde fashion via the abdominal aorta with $25 \mathrm{ml}$ of heparinized saline solution. Renal tissue was removed and coronal sections snap-frozen, stored at $-80^{\circ} \mathrm{C}$ and immersed in $10 \%$ neutral buffered formalin overnight and then paraffin-embedded.

\section{Creatinine Assay}

Plasma samples $(0.5 \mathrm{ml})$ were mixed with an equal volume of $5 \%$ trichloroacetic acid and then centrifuged at 5,000 rpm for 10 min. Urine samples were diluted 1:20 with $\mathrm{H}_{2} \mathrm{O}$ before assaying. A modified Jaffe reaction was used to measure creatinine levels by spectrophotometry at $510 \mathrm{~nm}$.

Arginine-Vasopressin ELISA

The assay used determined free AVP levels in plasma based on the competition between free AVP and an AVP tracer (AVP linked to acetylcholinesterase) for a limited number of AVPspecific rabbit antiserum binding sites. Colorimetric development of the reaction was carried out using Ellman's Reagent as a substrate for acetylcholinesterase. Log AVP levels were calculated from a standard curve derived from sample absorbance readings at $412 \mathrm{~nm}$. 


\section{Apoptag in situ Detection of Apoptosis}

Hydrated sections of rat kidney were digested with proteinase $\mathrm{K}$ and exposed to $3 \%$ hydrogen peroxide in methanol to quench endogenous peroxidase activity. Digoxigenin-labeled nucleotides were then added to free $3^{\prime}-\mathrm{OH}$ DNA termini in situ at $37^{\circ} \mathrm{C}$ by terminal deoxynucleotidyl transferase. Fragments thus labeled were detected using an anti-digoxigenin antibody conjugated to horseradish peroxidase with diaminobenzidine substrate. Sections were counterstained with Harris hematoxylin, dehydrated and coverslipped with DPX mounting medium.

\section{Urinary and Plasma Osmolality}

The osmolalities of plasma and urine samples were measured on an ADVANCE (Systems) Micro Osmometer 3,300, using depression of freezing point.

\section{Tubular Areas}

Hematoxylin and eosin-stained coronal sections of rat kidney were captured using the Aperio ScanScope CS system (Aperio, USA). Distal tubules in transverse section were identified by their relative sparsity and bulging nuclei in cortical areas. The circumferences of ten distal tubular segments per sample were highlighted and the luminal area in $\mu \mathrm{m}^{2}$ calculated. Mean areas per sample were calculated and used to generate group means.

\section{Protein Extraction and Western Blotting}

Powdered renal tissue (200 mg) was homogenized in $2 \mathrm{ml}$ tissue lysis buffer (140 mM NaCl, 10 mM EDTA, 10\% glycerol, 1\% NP40, 20 mM Tris ( $\mathrm{pH} 8.0$ ), $10 \mathrm{mM}$ phenylmethylsulphonyl fluoride, $1 \mathrm{~mm}$ leupeptin, $1 \mathrm{~mm}$ aprotinin, and $2 \mathrm{~mm}$ pepstatin) using a polytron homogenizer. Homogenates were lysed on ice for 30 min and centrifuged $\left(13,000 \mathrm{rpm}\right.$ at $\left.4^{\circ} \mathrm{C}\right)$. Supernatant protein concentrations were determined using a modified Lowry assay kit (Bio-Rad, UK).

Protein samples $(50 \mu \mathrm{g})$ were resolved in SDS polyacrylamide gels for $80 \mathrm{~min}$ at $140 \mathrm{~V}$, then electrophoretically transferred to a PDVF membrane at $100 \mathrm{~V}$ for $80 \mathrm{~min}$.

Membranes were incubated in blocking buffer ( $5 \%$ bovine serum albumin, BSA) in TTBS (Tris-buffered saline, $\mathrm{pH} 7.4,0.1 \%$ Tween 20) for $1 \mathrm{~h}$ at room temperature, then primary antibody $(1: 1,000)$ for a further $1 \mathrm{~h}$ in $3 \%$ BSA in TTBS. After washing for $5 \times 5 \mathrm{~min}$ in TTBS buffer, membranes were incubated for $1 \mathrm{~h}$ at room temperature with horseradish peroxidase-conjugated antirabbit IgG $(1: 5,000)$. Blots were further washed and then developed on SuperX X-ray film using enhanced chemiluminescent substrate. Scanning densitometry of developed blots was carried out using UN-SCAN-IT (Silk Scientific Corp., USA). Membranes were stained with Coomassie blue to check for transfer efficacy.

\section{RNA Extraction and cDNA Preparation}

Total RNA was extracted from powdered rat renal medulla specimens using Tri-Reagent and cDNA generated by reverse transcription of $1 \mu \mathrm{g}$ of total RNA using random hexamers following DNase digestion. RNA concentration and purity were measured by UV spectrophotometry.

V2R Expression in Renal Tissue

A V2R master mix was made which contained complete PCR mix (Promega) and $5 \mu \mathrm{M}$ each of V2 forward primer (5'AAGTCCTGCAGATGGTGGG-3') and V2R reverse primer ( $5^{\prime}$ -
Table 1. Plasma creatinine and creatinine clearance in male and female $\mathrm{UUO} / \mathrm{RN}$ rats $(\mathrm{n}=5)$

\begin{tabular}{llc}
\hline & \multicolumn{2}{l}{$\mathrm{UUO} / \mathrm{RN}$} \\
\cline { 2 - 3 } & male & female \\
\hline Plasma creatinine, $\mu \mathrm{M}$ & $87.6 \pm 20.5^{*}$ & $58.4 \pm 21.4$ \\
Creatinine clearance, $\mathrm{ml} / \mathrm{min} / 100 \mathrm{~g}$ & $0.3(0.1-0.4)^{*}$ & $0.6(0.3-1.3)$
\end{tabular}

Plasma creatinine values are expressed as mean \pm SD and creatinine clearance values as median (range).

${ }^{*} \mathrm{p}<0.05$, male UUO/RN vs. female UUO/RN rats.

ATCCAGGTGACATAGGCTCG-3'). A GAPDH master mix was likewise prepared to contain $5 \mu \mathrm{M}$ each of GAPDH forward and reverse primers (predefined assay, Applied Biosystems). The cycling conditions for both V2R and GAPDH were as follows: $95^{\circ} \mathrm{C}$ for $2 \mathrm{~min}, 30$ cycles; $95^{\circ} \mathrm{C}$ for $30 \mathrm{~s} ; 55^{\circ} \mathrm{C}$ for $30 \mathrm{~s} ; 72^{\circ} \mathrm{C}$ for $30 \mathrm{~s}$. Final elongation was carried out at $72^{\circ} \mathrm{C}$ for $10 \mathrm{~min}$ to allow final extension and samples stored at $-20^{\circ} \mathrm{C}$. Products were visualized using ethidium bromide gel electrophoresis and images were captured using UV illumination with Gel Capture software (AMPL Software, Australia). Relative densitometry was carried out on the bands using Gel Quant software (AMPL Software).

\section{Statistical Analysis}

Parametric data were expressed as mean \pm SD and compared by one-way ANOVA with Scheffe's post-hoc testing. Nonparametric data were expressed as median and interquartile range and compared by Kruskal-Wallis analysis. Statistical significance was set at $\mathrm{p}<0.05$.

\section{Results}

\section{Histology and Tubular Dilatation}

Distal tubules were markedly dilated in UUO/RN animals of both sexes (fig. 1a). The mean distal tubular area in male $\mathrm{UUO} / \mathrm{RN}$ rats was 2-fold higher than in male $\mathrm{S} / \mathrm{RN}$ rats $\left(1,048.17 \pm 124.48\right.$ vs. $521.53 \pm 100.71 \mu \mathrm{m}^{2}$; $\mathrm{p}<0.05)$. Mean distal tubular area in female UUO/RN rats was 1.6-fold higher than in female $\mathrm{S} / \mathrm{RN}$ rats $(639.45$ \pm 37.26 vs. $391.55 \pm 29.2 \mu \mathrm{m}^{2}$ ). Furthermore, the mean distal tubular area in male UUO/RN rats was 1.6-fold higher than in female UUO/RN rats (fig. 1b).

\section{Plasma Creatinine and Creatinine Clearance}

Plasma creatinine levels in male UUO/RN animals were 1.5-fold higher than in female UUO/RN animals (87.6 \pm 20.5 vs. $58.46 \pm 21.4 \mu \mathrm{M} ; \mathrm{p}<0.05$; table 1 ). Consequently, male UUO/RN creatinine clearance rates were 2-fold lower than in female UUO/RN rats ( $<<0.05$; table 1$)$. 

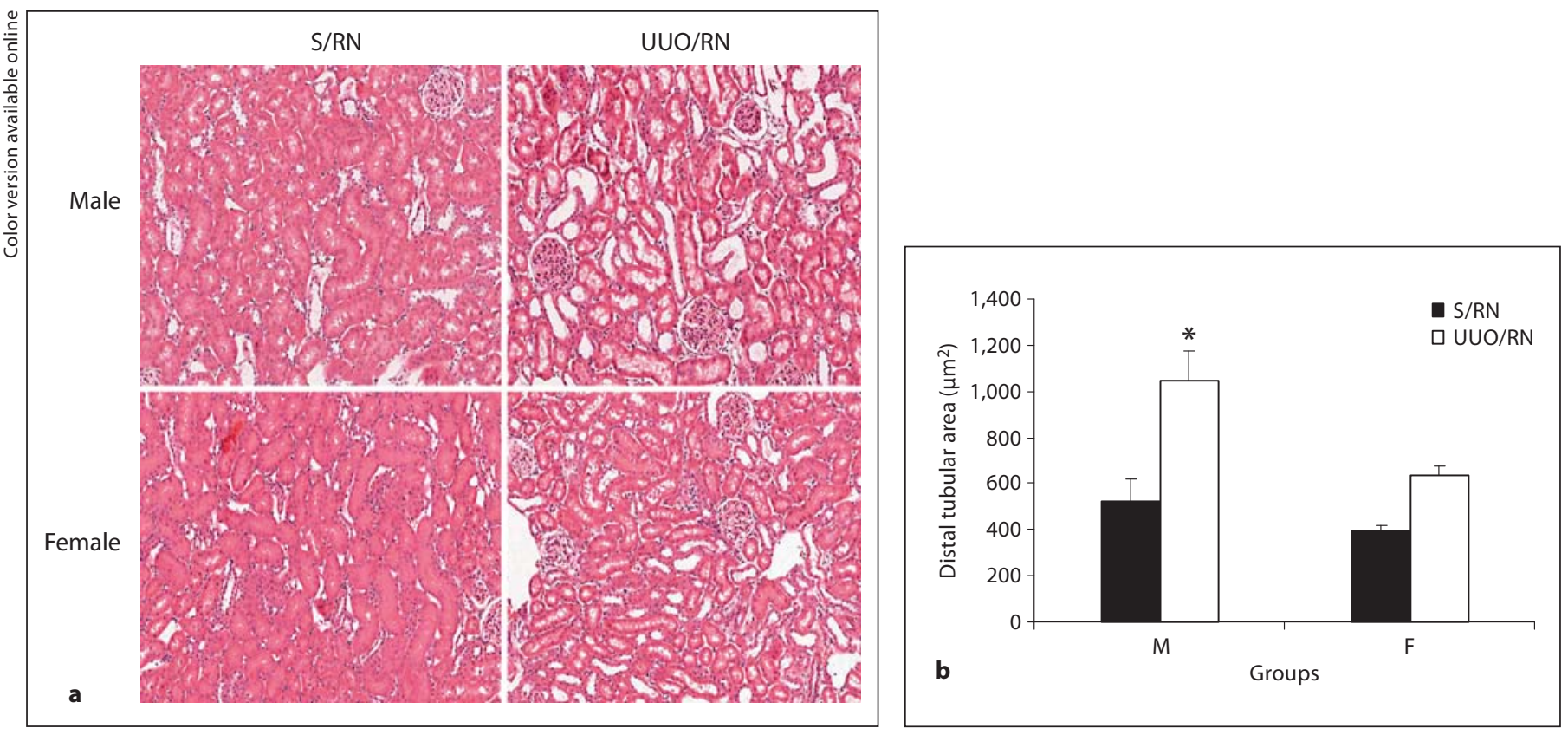

Fig. 1. Tubular dilatation following $S / R N$ and UUO/RN. a Low-power light micrographs $(\times 10)$ showing hematoxylin and eosin staining of renal cortical morphology 6 days after S/RN or UUO/RN in male and female rats. b Mean distal tubular area in S/RN and UUO/RN kidneys from male $(\mathrm{M})$ and female $(\mathrm{F})$ rats. Ten readings were taken from each of 3 male $\mathrm{S} / \mathrm{RN}$ and 3 male UUO/RN animals and 2 animals from each female group. ${ }^{*} \mathrm{p}<$ 0.05 , male $\mathrm{UUO} / \mathrm{RN}$ vs. male $\mathrm{S} / \mathrm{RN}$ rats.

\section{Tubular Apoptosis}

Cleaved caspase 3 levels were elevated in UUO/RN vs. $\mathrm{S} / \mathrm{RN}$ in male rats. Densitometric analysis of cleaved caspase 3 protein expression showed a 1.7-fold elevation in male UUO/RN kidneys vs. female UUO/RN kidneys $\left(14.2 \times 10^{4} \pm 3.7\right.$ vs. $8.4 \times 10^{4} \pm 1.0$; $\mathrm{p}<0.05$; fig. $\left.2 \mathrm{a}\right)$.

Apoptag quantification of apoptotic nuclei showed that UUO/RN male animals had twice as many apoptotic nuclei as UUO/RN females (3.6 apoptotic nuclei \pm 0.9 for $\mathrm{UUO} / \mathrm{RN}$ males vs. $1.8 \pm 0.6$ for $\mathrm{UUO} / \mathrm{RN}$ females; $\mathrm{p}<0.05$; fig. $2 \mathrm{~b}$ ).

\section{Urinary Volume and Osmolality before and after \\ Water Deprivation}

No significant differences were observed in urinary volume across groups during days 5-6. Animals in each group reduced their urine output during the second period (day 6-7) when $\mathrm{H}_{2} \mathrm{O}$ was completely restricted. Male S/RN day 6-7 urine output was 2.1-fold less than male S/RN day 5-6 output $(\mathrm{p}<0.01)$. Female $S / R N$ day $6-7$ output was 1.7 -fold less than female $\mathrm{S} / \mathrm{RN}$ day $5-6$ output $(p<0.05)$. Urinary output for male $U U O / R N$ animals during day 6-7 was 1.6 -fold lower than during day 5-6 $(\mathrm{p}<0.05)$, and for female UUO/RN animals in day 6-7 was 2.2 -fold less than in day $5-6$ ( $p<0.05$; fig. 3a).

On day 5-6, animals in UUO/RN groups showed a trend towards lower mean urinary osmolality than corresponding sex-matched S/RN animals. All groups had an increased urinary osmolality during the second period (day 6-7) when $\mathrm{H}_{2} \mathrm{O}$ was restricted, the increment being more notable in the UUO/RN groups. Female UUO/ $\mathrm{RN}$ animals had a urinary osmolality 1.4 -fold higher during day $6-7$ than day $5-6(1,901 \pm 173$ vs. $1,340 \pm 460$ mosm, $\mathrm{p}<0.05)$. Male $\mathrm{UUO} / \mathrm{RN}$ rats' urinary osmolality was 1.3-fold higher during day 6-7 than day 5-6 (1,768 \pm 237 vs. $1,350 \pm 428$ mosm; $\mathrm{p}<0.05$; fig. $3 \mathrm{~b}$ ).

\section{Plasma AVP and Renal Medullary V2R and AQP2}

Plasma AVP levels were not significantly different between UUO/RN and S/RN rats of either sex. However, in both sexes, the data were skewed towards higher values. No significant difference was observed in the median values of plasma AVP between male and female UUO/RN rats (fig. 4a). 
Fig. 2. Apoptosis after UUO/RN. a Western blot for cleaved caspase 3 in male and female S/RN and UUO/RN animals. Scanning densitometry of the above blot quantifying cleaved caspase 3 protein expression ( $\mathrm{n}=2$ for S/RN animals, $\mathrm{n}=3$ for $\mathrm{UUO} / \mathrm{RN}$ animals) is shown underneath. ${ }^{*} \mathrm{p}<0.05$, male UUO/RN vs. female UUO/RN rats. b High-power field $(\times 20)$ micrographs of sections of male and female UUO/RN rats after staining of apoptotic nuclei with Apoptag. For each group, ten random cortical readings $\left(\times 10\right.$ power) were taken for 3 animals $(n=3) .{ }^{*} \mathrm{p}<0.05$, male $\mathrm{UUO} / \mathrm{RN}$ vs. female $\mathrm{UUO} / \mathrm{RN}$ rats.
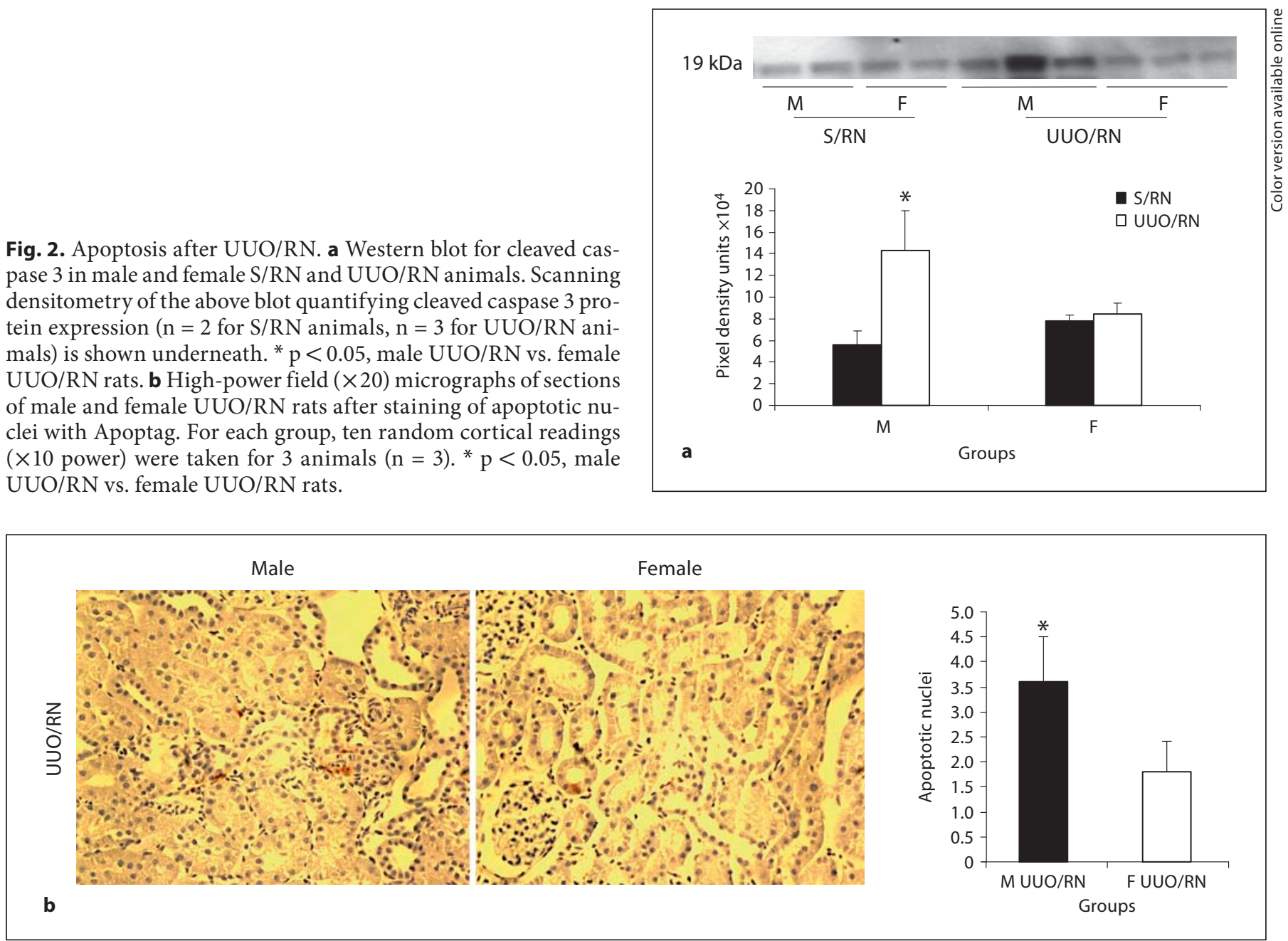

No statistically significant alteration between UUO/ $\mathrm{RN}$ male and female animals was observed for V2R expression (fig. 4b).

Female S/RN animals had a 2.9-fold lower protein expression for AQP2 than their male counterparts (10.3 \pm 4.3 vs. $30.04 \pm 0.38$ ). Female UUO/RN rats had a 2.68 fold higher expression of AQP2 protein than female S/RN rats $(27.7 \pm 3.116$ vs. $10.3 \pm 4.3)$, while no difference was discernable in AQP2 levels between male S/RN and $\mathrm{UUO} / \mathrm{RN}$ animals $(30.04 \pm 0.38$ vs. $33.2 \pm 1.8$; fig. $4 \mathrm{c})$.

\section{Discussion}

The results show that male gender is associated with a worsened GFR and a higher tubular apoptotic index. However, no significant differences were observed with regard to the volume or osmolality of the urine produced by the postobstructed kidney or the ability to concentrate urinary output in the face of $\mathrm{H}_{2} \mathrm{O}$ restriction. Analysis of the constituent components of the AVP-V2R-AQP2 axis demonstrates that AQP2 expression is increased in the postobstructive period in both sexes but that the increase in males is of a much smaller magnitude due to an apparently higher generalized baseline male gender-associated AQP2 expression.

The evidence of a significant increase in plasma creatinine and the associated decrease in creatinine clearance rates point to a sustained reduction in GFR following relief of obstruction in male rats. Obstructive injury is associated with reductions in GFR, and Metcalfe et al. $[13,14]$ have noted that only male rats show a reduction in GFR following 3 days of sustained UUO. Endothelin (ET) is an androgen-inducible downstream component of the RA 


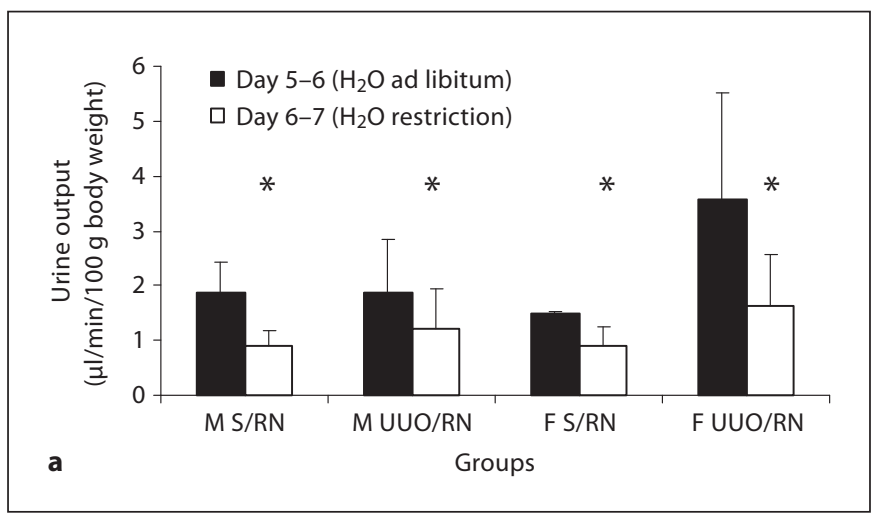

Fig. 3. Urinary volume and osmolality after UUO/RN before and after water restriction. a Volume of urine excreted by male and female S/RN and UUO/RN rats in the first $24 \mathrm{~h}$ (day 5-6; period of access to water $\left(\mathrm{H}_{2} \mathrm{O}\right)$ ad libitum) and second $24 \mathrm{~h}$ (day 6-7; period of total water restriction) in metabolic cages $(n=5) .{ }^{*} \mathrm{p}<$ 0.05 , day 6-7 sample vs. cognate day $5-6$ sample. b Urinary osmo-

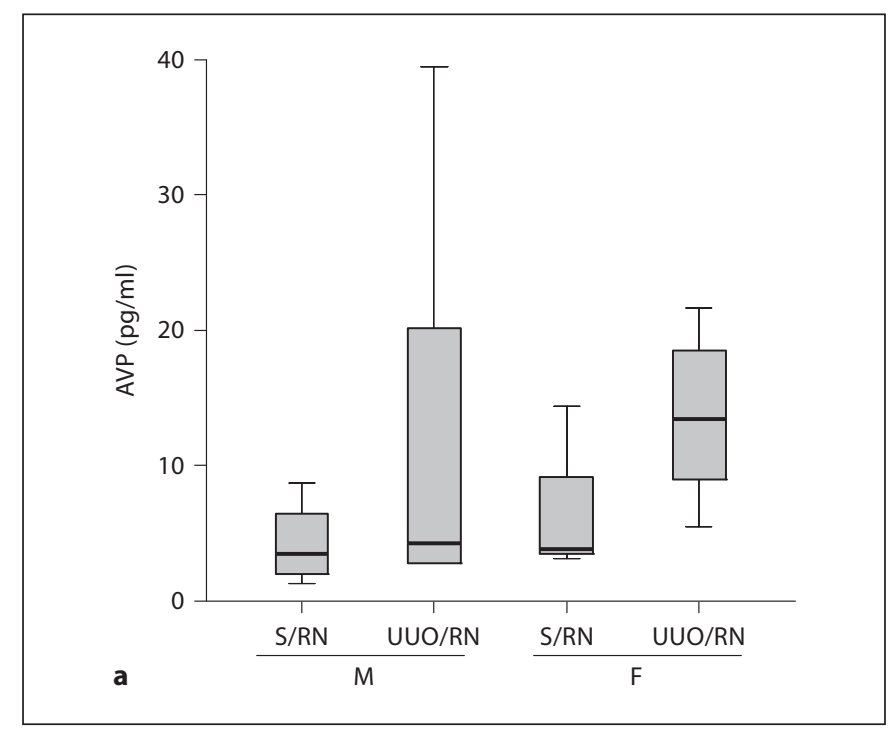

Fig. 4. Plasma AVP, renal V2R expression and AQP2 protein levels in $\mathrm{S} / \mathrm{RN}$ and $\mathrm{UUO} / \mathrm{RN}$ animals after water restriction. a Plasma AVP levels in male and female S/RN and UUO/RN rats. b Renal V2R expression level as assessed by RT-PCR in male and female $\mathrm{S} / \mathrm{RN}$ and UUO/RN rats with a positive control (PC) of MadinDarby collecting duct cell RNA extract also included. The corresponding graph underneath shows the relative quantity (RQ) of V2R mRNA as a percentage of GAPDH. c Western blot for whole medullary AQP2 in male and female S/RN and UUO/RN rats. Scanning densitometry of the above blot quantifying AQP2 protein expression is shown underneath.

Gender Effects in the Postobstructive Kidney

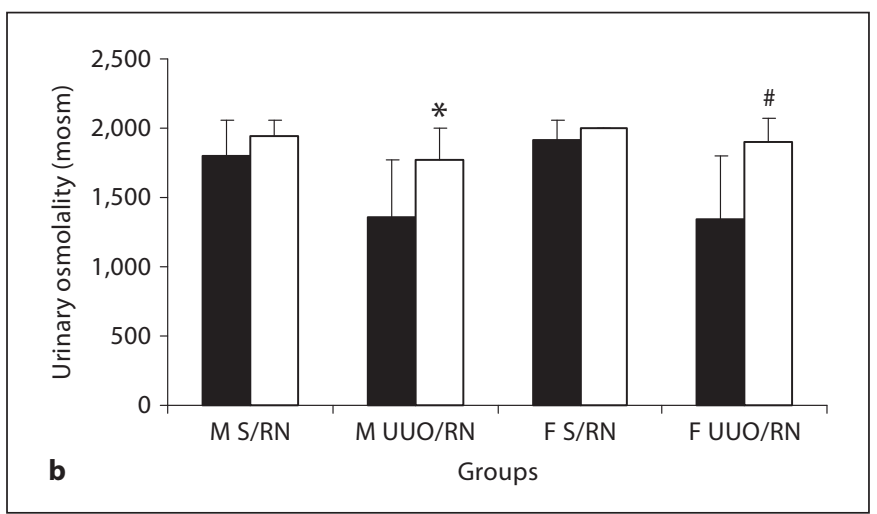

lality in male and female $\mathrm{S} / \mathrm{RN}$ and $\mathrm{UUO} / \mathrm{RN}$ rats in the first $24 \mathrm{~h}$ (day 5-6; period of access to water ad libitum) and second $24 \mathrm{~h}$ (day 6-7; period of total water restriction) in metabolic cages $(\mathrm{n}=5) .{ }^{*} \mathrm{p}<0.05$, male UUO/RN day 6-7 sample vs. cognate day 5-6 sample; ${ }^{*} \mathrm{p}<0.05$, female $\mathrm{UUO} / \mathrm{RN}$ day $6-7$ sample vs. cognate day 5-6 sample.
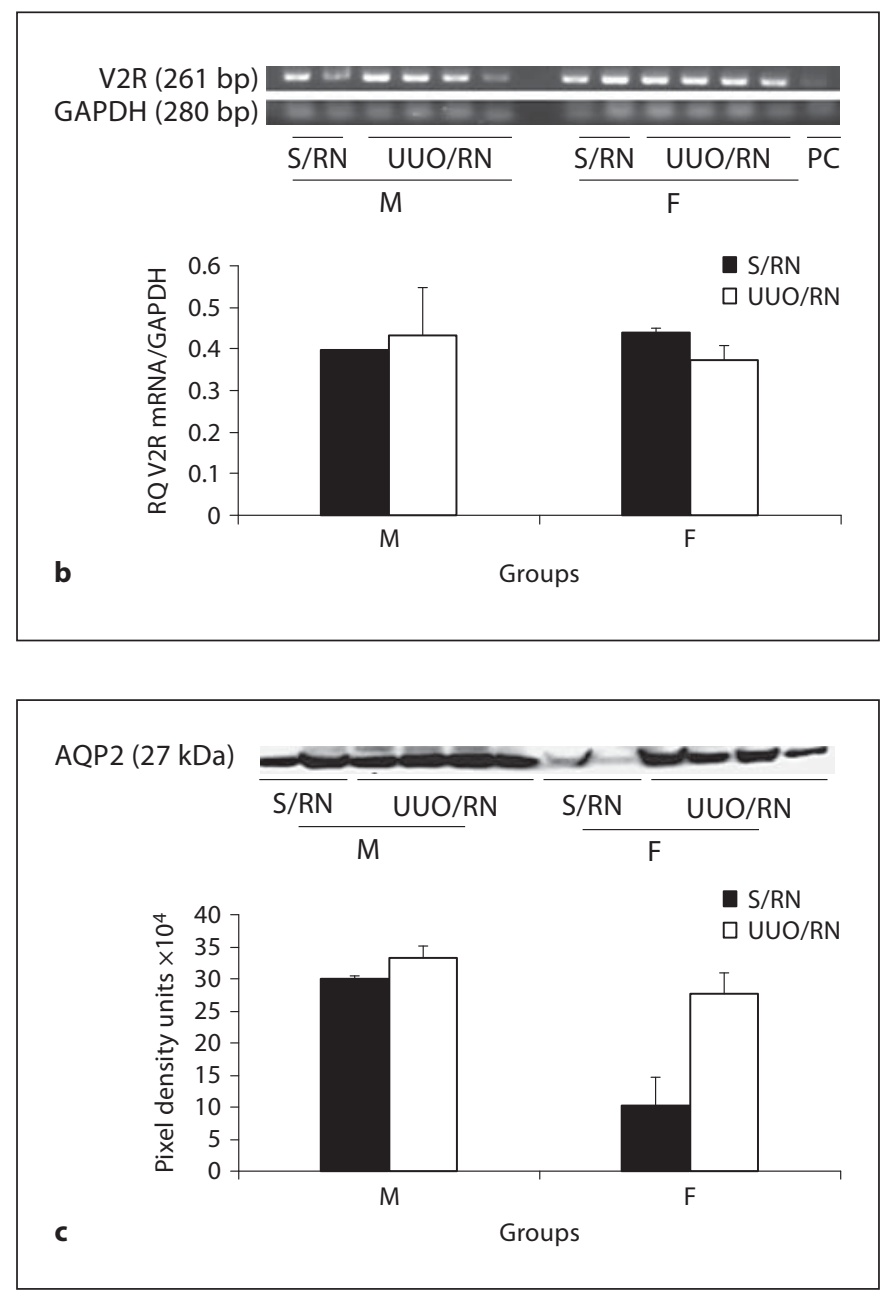

Kidney Blood Press Res 2010;33:266-273 
system which, via engagement of type A (ET-A) receptors, leads to constriction of the afferent and efferent glomerular arteriolae, thereby reducing renal blood flow (RBF) and GFR [15-17]. ET levels are increased in the postobstructive kidney [16] and have been shown to be more sharply increased in the male rat kidney following renal ischemia-reperfusion injury [11]. Pretreatment of rats with an ET-A antagonist improves RBF following UUO [16] and prevents the sex-dependent increase in postischemic mortality observed in male rats [11]. Therefore, elevation of components of the intra-renal RA system may account for the decrease in GFR in male rats observed in this study.

Male rats showed an increase in renal active caspase-3 levels and tubular apoptosis. This is in agreement with an increase in tubular apoptosis in male rats following 3 days of sustained UUO in previous studies [13]. Testosterone has been reported to have a direct proapoptotic effect in vitro in proximal tubular cell cultures [18]. However, the expression of a range of renal transporters operative in normal renal function (e.g. the $\mathrm{Na}^{+} / \mathrm{H}^{+}$antiporter [19]) is positively modulated by androgens, and therefore a proapoptotic effect per se of androgens in the normal kidney appears unlikely. Rather, via the activation of mediators that promote reduced renal perfusion, oxidative stress and inflammation, it is likely that the increased apoptosis observed in male rats is a result of a stimulatory effect of androgens on the expression/activity of a number of injury-associated factors culminating in increased tubular cell stress and hence apoptosis [10]. This hypothesis is supported by the observation that antagonism of the ET-A receptor, in addition to normalizing RBF, also diminishes the apoptotic response to UUO of 24 -hour duration in male rats [16].

It might be expected that these results would translate into an observable difference in the grade of postobstructive diuresis between male and female rats. The results show that there is no significant difference in urinary output or urinary osmolality between sham and obstructed rats of either sex. The results do however clearly show a definite tendency for reduced urinary osmolality in the postobstructed groups vs. sham. Obstructed rats of each sex retain the ability to significantly concentrate urine in response to $\mathrm{H}_{2} \mathrm{O}$ restriction. These data support the conclusion that the postobstructed kidneys of both sexes retain an adaptive function in spite of an observable increase in mild grade injury in the male postobstructed kidney.

The question therefore arises as to why postobstructive diuresis is not more prominent in male rats. Clearly, the reduction in GFR in male rats has the potential to limit a polyuric response, and this may account in part for the findings. Additionally, we investigated whether perhaps an enhanced sensitivity of the AVP-V2R-AQP2 axis may act to offset the propensity for increased postobstructive diuresis in males. An enhanced AVP release in males in response to hypertonic saline infusion has been demonstrated in humans [20]. However, our results do not support the hypothesis that the discord between renal injury and grade of diuresis was due to an enhanced central sensitivity to urinary free $\mathrm{H}_{2} \mathrm{O}$ clearance.

Angiotensin II and ET-1 have been shown to induce the expression of the V2R in the inner medullary collect duct in the rat [21]. Therefore, we studied the mRNA expression of V2R in extracts of male and female postobstructed rats. However, no significant difference was observed, and hence V2R expression in the male postobstructed kidney did not appear to be involved in enhancing sensitivity of the male kidney to AVP and thereby limiting diuresis.

Male gender was associated with an increase in the expression of AQP2. Interestingly, in the experiments presented here, AQP2 levels were increased in postobstructed animals, in contrast to previous reports showing that sustained obstruction and resultant diuresis are associated with downregulated renal AQP expression [8]. The results herein suggest that in the postobstructive setting, animals are subject to a sustained challenge with regard to $\mathrm{H}_{2} \mathrm{O}$ conservation, and AQP2 upregulation constitutes a component of the homeostatic response to this. A role for the androgen-RA axis leading to an increased magnitude in this response is supported by the observation that renal AQP2 upregulation in the rat following AVP infusion is blocked by the addition of the angiotensin II type 1 receptor antagonist candesartan [22]. In summary, the work presented demonstrates persistence of a mild but quantifiable impairment in renal structure and function 1 week after the release of an acute UUO of 24-hour duration in male rats. This is not associated with a worsening of postobstructive diuresis, possibly a function of the reduction in GFR and a salutary effect of increased AQP2 expression.

\section{Acknowledgements}

The authors acknowledge the support of the U.C.D. Biomedical Facility, Department of Clinical Biochemistry, Beaumont Hospital, and the advice of Prof. C. Thompson (R.C.S.I.). M.R.Q. was in receipt of a research grant from the British Urological Foundation. 


\section{References}

1 Thulesius O, Angelo-Khattar M, Sabha M: The effect of ureteral distension on peristalsis. Studies on human and sheep ureters. Urol Res 1989;17:385-388.

$\checkmark 2$ Lennon GM, Ryan PC, Fitzpatrick JM: Recovery of ureteric motility following complete and partial ureteric obstruction. Br J Urol 1993;72:702-707.

-3 Quinlan MR, Wright E, Dillon-Murphy R, Watson RWG, Fitzpatrick JM, Docherty NG: Analysis of urinary and plasma electrolytes in a rat model of unilateral ureteric obstruction (UUO). Open Urol Nephrol J 2008;1: $16-21$.

4 Quinlan MR, Docherty NG, Watson RW, Fitzpatrick JM: Exploring mechanisms involved in renal tubular sensing of mechanical stretch following ureteric obstruction. Am J Physiol Renal Physiol 2008;295:F1-F11.

$\checkmark 5$ Docherty NG, O’Sullivan OE, Healy DA, Fitzpatrick JM, Watson RW: Evidence that inhibition of tubular cell apoptosis protects against renal damage and development of fibrosis following ureteric obstruction. Am J Physiol Renal Physiol 2006;290:F4-F13.

$\checkmark 6$ Valles P, Merlo V, Beron W, Manucha W: Recovery of distal nephron enzyme activity after release of unilateral ureteral obstruction. J Urol 1999;161:641-648.

7 Kim SW, Lee J, Jung K, Ma SK, Oh Y, Kim WY, Choi KC, Kim J: Diminished expression of sodium transporters in the ureteral obstructed kidney in rats. Nephron Exp Nephrol 2004;96:e67-e76.

$>8$ Li C, Wang W, Knepper MA, Nielsen S, Frokiaer J: Downregulation of renal aquaporins in response to unilateral ureteral obstruction. Am J Physiol Renal Physiol 2003; 284:F1066-F1079.
9 Li C, Klein JD, Wang W, Knepper MA, Nielsen S, Sands JM, Frokiaer J: Altered expression of urea transporters in response to ureteral obstruction. Am J Physiol Renal Physiol 2004;286:F1154-F1162.

10 McGuire BB, Watson RW, Perez-Barriocanal F, Fitzpatrick JM, Docherty NG: Gender differences in the renin-angiotensin and nitric oxide systems: relevance in the normal and diseased kidney. Kidney Blood Press Res 2007;30:67-80.

11 Muller V, Losonczy G, Heemann U, Vannay A, Fekete A, Reusz G, Tulassay T, Szabo AJ: Sexual dimorphism in renal ischemia-reperfusion injury in rats: possible role of endothelin. Kidney Int 2002;62:1364-1371.

12 Antus B, Yao Y, Liu S, Song E, Lutz J, Heemann U: Contribution of androgens to chronic allograft nephropathy is mediated by dihydrotestosterone. Kidney Int 2001;60: 1955-1963.

13 Metcalfe PD, Leslie JA, Campbell MT, Meldrum DR, Hile KL, Meldrum KK: Testosterone exacerbates obstructive renal injury by stimulating TNF-alpha production and increasing proapoptotic and profibrotic signaling. Am J Physiol Endocrinol Metab 2008;294:E435-E443.

14 Wahlberg J: The renal response to ureteral obstruction. Scand J Urol Nephrol Suppl 1983;73:1-30.

15 Polderman KH, Stehouwer CD, van Kamp GJ, Dekker GA, Verheugt FW, Gooren LJ: Influence of sex hormones on plasma endothelin levels. Ann Intern Med 1993;118:429432 .
16 Hegarty NJ, Young LS, O’Neill AJ, Watson RW, Fitzpatrick JM: Endothelin in unilateral ureteral obstruction: vascular and cellular effects. J Urol 2003;169:740-744.

17 Cairns HS, Rogerson ME, Fairbanks LD, Neild GH, Westwick J: Endothelin induces an increase in renal vascular resistance and a fall in glomerular filtration rate in the rabbit isolated perfused kidney. Br J Pharmacol 1989;98:155-160.

$>18$ Verzola D, Gandolfo MT, Salvatore F, Villaggio B, Gianiorio F, Traverso P, Deferrari G, Garibotto G: Testosterone promotes apoptotic damage in human renal tubular cells. Kidney Int 2004;65:1252-1261.

19 Quan A, Chakravarty S, Chen JK, Chen JC, Loleh S, Saini N, Harris RC, Capdevila J, Quigley R: Androgens augment proximal tubule transport. Am J Physiol Renal Physiol 2004;287:F452-F459.

-20 Stachenfeld NS, Splenser AE, Calzone WL, Taylor MP, Keefe DL: Sex differences in osmotic regulation of AVP and renal sodium handling. J Appl Physiol 2001;91:1893-1901.

21 Wang MH, Fok A, Huang MH, Wong NL: Interaction between endothelin and angiotensin II in the up-regulation of vasopressin messenger RNA in the inner medullary collecting duct of the rat. Metabolism 2007;56: 1372-1376

$\checkmark 22$ Kwon TH, Nielsen J, Knepper MA, Frokiaer J, Nielsen S: Angiotensin II AT1 receptor blockade decreases vasopressin-induced water reabsorption and AQP2 levels in $\mathrm{NaCl}$ restricted rats. Am J Physiol Renal Physiol 2005;288:F673-F684. 